\title{
Four generalized Weibull distributions: Similar properties and applications
}

\author{
Edwin Ortega '; Fábio Prataviera I"; Gauss Moutinho Cordeiro III
}

\begin{abstract}
We derive a common linear representation for the densities of four generalizations of the two-parameter Weibull distribution in terms of Weibull densities. The four generalized Weibull distributions briefly studied are: the Marshall-Olkin-Weibull, betaWeibull, gamma-Weibull and Kumaraswamy-Weibull distributions. We demonstrate that several mathematical properties of these generalizations can be obtained simultaneously from those of the Weibull properties. We present two applications to real data sets by comparing these generalized distributions. It is hoped that this paper encourage developments of further generalizations of the Weibull based on the same linear representation.
\end{abstract}

Keywords: Beta Weibull; Gamma-Weibull; Kumaraswamy-Weibull; Marshall-OlkinWeibull 


\section{INTRODUCTION}

The two-parameter Weibull distribution is named after Waloddi Weibull pioneered this distribution to model data sets of widely differing characteristics. Over the last three decades many Weibullrelated distributions have been constructed with a view for applications in various areas such as medicine, reliability, engineering, survival analysis, demography, actuarial study and several others. The Weibull distribution has been so far the most important distribution for modeling lifetime data and phenomenon with monotone hazard rates. When modeling these types of hazard rates, it may be an initial choice because of its negatively and positively skewed density shapes. Its major weakness is its inability to accommodate nonmonotone hazard rates (in particular, bathtub shaped hazard rates) which has lead to seek for some of its generalizations.

The first generalization allowing for non-monotone hazard rates, including the bathtub shaped hazard rate, is the exponentiated Weibull (EW) distribution pioneered by Mudholkar and Srivastava (1993) which provides significantly better fits than traditional models based on the exponential, gamma, Weibull, log-logistic and lognormal distributions.

The cumulative distribution function (cdf) and probability density function (pdf) of the twoparameter Weibull distribution (for $x>0$ ) are

$$
\begin{aligned}
& G_{\lambda, c}(x)=1-\exp \left[-(\lambda x)^{c}\right] \\
& \text { and } \\
& g_{\lambda, c}(x)=c \lambda^{c} x^{c-1} \exp \left[-(\lambda x)^{c}\right]
\end{aligned}
$$

respectively, where $c>0$ is a shape parameter and $\lambda>0$ is a scale parameter. Henceforth, we write $T \sim W(\lambda, c)$ for a random variable $T$ having density (2). The quantile function (qf) of $T$ is $Q \lambda, c(u)=G-\lambda, c 1(u)=\lambda-1[-\log (1-u)] 1 / c$.

The paper is unfolded as follows. In Section 2, we review four generalized Weibull distributions which have been investigated in the last twenty years based of 
the families introduced by Marshall and Olkin (1997), Eugene et al. (2002), Zografos and Balakrishnan (2009) and Cordeiro and de Castro (2011). We also give simple forms to generate data from these generalized models. In Section 3, we provide a common linear representation for the densities of the four generalized distributions in terms of Weibull densities. Some mathematical properties for all of them can be determined from this useful linear representation and those Weibull properties as shown in Section 4. Other extended Weibull distributions are addressed telegraphically in Section 5. Maximum likelihood estimation is summarized in Section 6. In Section 7, we compare the four generalized Weibull models by means of two real life data sets. Finally, Section 8 offers some concluding remarks.

\section{FOUR GENERALIZED WEIBULL DISTRIBUTIONS}

Henceforth, we consider the Weibull distribution as defined by (1) and (2) to present four generalized Weibull distributions.

The first generalization follows the Marshall and Olkin's (1997) method to expand a distribution by adding an extra shape parameter. There are more than 30 published papers on distributions generated using this method. The cdf of the Marshall-Olkin-Weibull (MOW) distribution (for $\theta>0$ ) can be expressed as

$$
F_{\text {MON }}(x)=\frac{G_{\lambda, c}(x)}{G_{\lambda, c}(x)+\theta\left[1-G_{\lambda, c}(x)\right]}, \quad x>0
$$

The quantile function ( $q f$ ) of the MOW distribution is easily determined as $Q_{\text {Mow }}(u)=Q_{\lambda, c}(\theta u /[1-(1-\theta) u])$. The density function corresponding to (3) takes the form

$$
f_{\text {MOW }}(x)=\frac{\theta g_{, c}(x)}{\left\{1-(1-\theta)\left[1-G_{h, c}(x)\right]\right\}^{2}}
$$

For $\theta=1, f_{\operatorname{MOW}}(x)$ is equal to $g_{\lambda, c}(x)$ and, for different values of $\theta, f_{\operatorname{MOW}}(x)$ can be more flexible than $g_{\lambda, c}(x)$. 
The hazard rate function (hrf) of the MOW distribution reduces to

$$
I_{\text {MOW }}(x)=\frac{T_{\lambda, c}(x)}{1-(1-\theta)\left[1-G_{\lambda, c}(x)\right]} \text {, }
$$

where $\tau_{\lambda, c}(x)$ is the Weibull hrf corresponding to (2).

The last equation implies that $\tau_{\operatorname{MOW}}(x) / \tau_{\lambda, c}(x)$ is increasing in $x$ for $\theta>1$ and decreasing in $x$ for $0<\theta<1$. So, the extra parameter $\theta$ is called "tilt parameter", since the hrf of the MOW distribution is shifted below $(\theta>1)$ or above $(0<\theta<1)$ of the Weibull hrf. In fact, for all $x>0, \tau_{\operatorname{Mow}}(x) \leq \tau_{\lambda, c}(x)$ when $\theta \geq 1$, and $\tau_{\operatorname{Mow}}(x) \geq \tau_{\lambda, c}(x)$ when $0<$ $\theta \leq 1$. Further details were addressed by Marshall and Olkin (1997).

The second generalization is the beta-Weibull (BW) distribution (with four positive parameters) which follows from the family pioneered by Eugene et al. (2002) by taking the Weibull as the baseline model. The BW cdf with two additional shape parameters $a>0$ and $b>0$ is

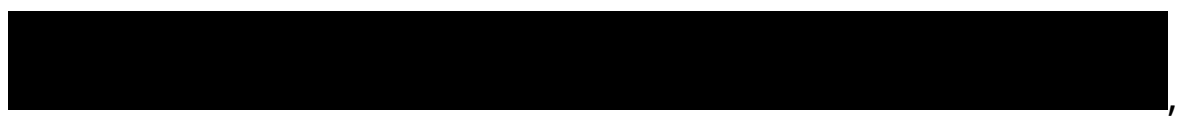

where $B(a, b)=\Gamma(a+b) /\lceil\Gamma(a) \Gamma(b)]$ is the beta function, $\Gamma($ is the incomplete beta gamma function and function ratio. The pdf corresponding to (5) has the form

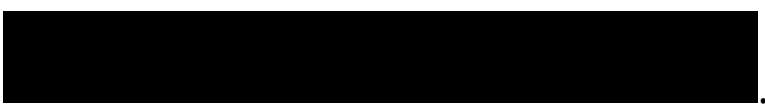

The BW distribution includes as special cases the EW $(b=1)$, exponentiated exponential (EE) $(b=c=1)$, Weibull $(a=b=1)$, exponential ( $a=b=c=1)$, Burr Type X and Type 2 extreme value distributions as well as the distribution of the order statistic from a Weibull population. Its applications have been widespread in oncology, finance, system failures, reliability prediction, extreme value data using floods, models for carbon fibrous composites, modeling tree diameters, among several others.

The pdf and the hrf of the BW distribution are obtained from (1), (2), (5) and (6) using $I_{x}(a, b)=I_{1-x}(b, a)$ as 


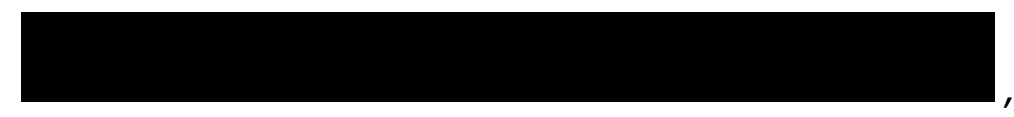

and

respectively.

The shape of $\tau_{\mathrm{BW}}(x)$ is constant when $a=c=1$, decreasing when $a c \leq 1$ and $c \leq 1$, increasing when $a c \geq 1$ and $c \geq 1$, bathtub when $a c<1$ and $c>1$, and unimodal when $a c>1$ and $c<1$.

The qf of the BW distribution follows easily from the beta qf. By inverting (5), we can write

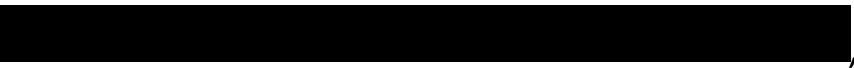

where $I_{u^{-}}{ }^{1}(a, b)$ denotes the inverse of the incomplete beta function ratio. Some expansions for

$I_{u^{-}}{ }^{1}(a, b)$ can be found in Wolfram website ${ }^{1}$. So, we can generate BW random variables by

$$
X=\lambda-{ }^{1}\{-\log (1-V)\}^{1 / c},
$$

where $V$ is a beta variate with shape parameters $a$ and $b$.

The third generalization follows from Zografos and Balakrishnan's (2009) gamma family with an extra shape parameter $a>0$. The cdf of the gamma-Weibull (GW) distribution is

$$
F_{G W}(x)=\frac{Y\left(a_{s}-\log \left[1-G_{h, c}(x)\right]\right)}{\Gamma(a)}=\frac{1}{\Gamma(a)} \int_{0}^{\log \left[1-G_{h, c}(x)\right]} t^{a-1} e^{-t} d t
$$

where $\quad t^{t} d t$ is the lower incomplete gamma function. The GW density takes the

form

\footnotetext{
${ }^{1}$ http://functions.wolfram.com/06.23.06.0004.01
} 


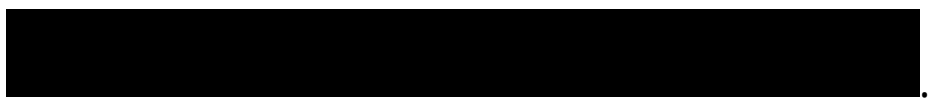

A physical motivation for the GW distribution can follow from Zografos and Balakrishnan (2009): if $X_{L(1), \ldots, X_{L(n)}}$ are lower record values from a sequence of independent random variables with common Weibull pdf $g_{\lambda, c}(x)$, then the pdf of the $n$th lower record value has the form (8). If $Z$ is a gamma random variable with unit scale parameter and shape parameter $a>0$, then $X=Q_{\lambda, c}\left(\mathrm{e}^{\mathrm{z}}\right)$ has density (8). So, the GW distribution is easily generated from the gamma distribution and the Weibull qf.

Further, the GW density function (for $x>0$ ) can be expressed as

$$
f_{\mathrm{GW}}(x)=\frac{c \lambda^{c a}}{\Gamma(a)} x^{a c-1} \exp \left\{-(\lambda x)^{c}\right\}
$$

Equation (9) extends some distributions previously discussed in the literature. In fact, it is identical to the generalized gamma distribution (Stacy, 1962). The Weibull distribution is a basic exemplar when $a=1$, whereas the gamma distribution follows when $c=1$. The half-normal distribution is obtained for $a=3$ and $c=2$. In addition, the log-normal distribution is a limiting special case when $a$ tends to infinity.

By inverting (7), the qf of the GW distribution follows as

$$
\mathrm{Q}_{\mathrm{GW}}(\mathrm{u})=\mathrm{Q}_{\lambda, \mathrm{c}}\left(1-\exp \left[-\mathrm{Q}^{-1}(\mathrm{a}, 1-u)\right]\right)=\lambda^{-1}\left[\mathrm{Q}^{-1}(\mathrm{a}, 1-u)\right]^{1 / c}
$$

for $0<u<1$, where $Q{ }^{1}(a, u)$ is the inverse function of $Q(a, x)=1 \gamma(a, x) / \Gamma(a)$, see http:// functions.wolfram.com/ GammaBetaErf/ InverseGammaRegularized/ for details. Further, the simulation of the GW random variable is quite easy: if $V$ is a gamma random variable with shape parameter $a$ and unit scale parameter, then $X=$ $\lambda-{ }^{1} V^{1 / c}$ will have the density (9).

Finally, the fourth generalization is the Kumaraswamy-Weibull (KwW) distribution (with four positive parameters) defined from Cordeiro and de Castro's (2011) family by the cdf and pdf 
$F_{K W W}(x)=1-\left\{1-G_{h_{,}}(x)^{a}\right\}^{b}, \quad x>0$,

and

$f_{\text {Kaw }}(x)=a b g_{, C}(x) G_{h, c}(x)^{a-1}\left\{1-G_{h, c}(x)^{a}\right\}^{b-1}$,

respectively. Then, the KwW density (for $x>0$ ) follows from (1), (2) and (12) as

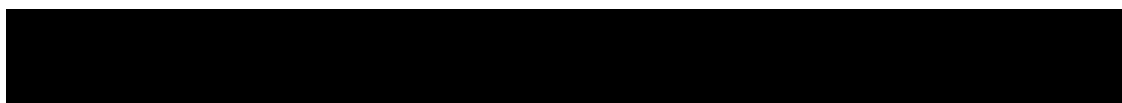

It contains as special cases the EW $(b=1)$, Kumaraswamy exponential $(c=1)$, exponentiated Rayleigh, exponentiated exponential, Weibull, Rayleigh and exponential distributions. It allows for bathtub shaped, monotonically decreasing, monotonically increasing, and upside down bathtub shaped hazard rates.

The role of the two extra parameters $a$ and $b$ is to govern skewness and obtain the KwW distributions with heavier or ligther tails than the Weibull distribution. In fact, the tails of $f_{\operatorname{kwW}}(x)$ will be lighter than those of $g_{\lambda, c}(x)$ if $a>1$ or $b>1$. On the other hand, the tails of $f_{\operatorname{kww}}(x)$ will be heavier than those of $g_{\lambda, c}(x)$ if $a<1$ or $b<1$.

The qf of the KwW distribution is readily obtained from the Weibull qf. In fact, the qf corresponding to (11) is

$$
\mathrm{Q}_{\mathrm{KwW}}(u)=\mathrm{Q}_{\lambda, \mathrm{c}}\left(\left[1-(1-u)^{1 \mathrm{t}}\right]^{1 \mathrm{a}}\right)
$$

Further, we can generate KwW variates by

$$
X=\lambda^{-1}\left[-\log \left\{1-\left[1-(1-U)^{1 / c}\right]^{1 / a}\right\}\right]^{1 s c}
$$

where $U$ is a uniform variate on the unit interval $(0,1)$. 


\section{LINEAR REPRESENTATIONS}

The four generalized Weibull densities defined previously can be written as a common linear combination of Weibull densities whose coefficients depend only on the parameters of the generator family.

The cdf and pdf of the EW distribution with power parameter $a>0$ have the forms
$\Pi \alpha, \lambda, c(x)=G \lambda, c(x) \alpha$
and
$\pi \alpha, \lambda, c(x)=\alpha G \lambda, c(x) \alpha-1 g \lambda, c(x)$,

respectively.

We can expand $\Pi a, \lambda, c(x)$ from (1) using the power series for real non-integer $a>$ 0

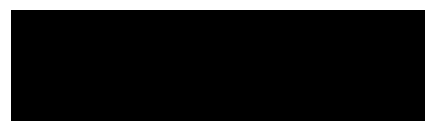

for $|z|<1$. Then, we can write

$\Pi_{a, \lambda, c}(x)=\sum_{j=0}^{\infty} v_{j}(\alpha) g \lambda_{j}, c(x)$

where and $g_{\lambda, c}(x)$ is the Weibull pdf (1) with scale parameter $\lambda_{j}$ $=(j+1)^{1 / c} \lambda$ and shape parameter $c$.

The following four linear representations for the generalized Weibull distributions described in Section 2 are derived by using the linear combinations of the family densities provided by some authors in conjunction with Equation (13). These linear representations for the four densities in terms of Weibull densities are important to determine their mathematical properties from those of the Weibull distribution.

First, based upon the general expansion by Cordeiro et al. (2014), the MOW density (4) can be expressed as 
$f_{\text {MOW }}(x)=\sum_{i=0}^{\infty} w_{i}^{M O W} T_{i+1, \lambda, c}(x)$

where the coefficients are (for $i=0,1, \ldots)$

$\mathbf{W}_{\mathbf{i}}^{\text {MOW }}=\mathbf{W}^{\text {MOW }}(\theta)= \begin{cases}\frac{(-1)^{i} \theta}{(i+1)} \sum_{k=i}^{\infty}\left(\begin{array}{c}k \\ i\end{array}\right)(k+1) \bar{\theta}^{k}, & \theta \in(0,1) \\ \theta^{-1}\left(1-\theta^{-1}\right)^{i}, & \theta>1,\end{cases}$

and $\theta^{-}=1-\theta$. By inserting (13) in the last expression for $f_{\mathrm{MOW}}(x)$ and changing the sums where $\delta_{j}=0$ for $j=0,1$ and $\delta_{j}=j-1$ for $j \geq 2$, we obtain

$f_{\text {MON }}(x)=\sum_{j=0}^{m} t_{j}^{\text {MON }} g_{h_{j}, c}(x)$,

where tmowi

Second, for the BW density (6), we obtain from Nadarajah et al. (2012)

$f_{B W}(x)=\sum_{i=0}^{\infty} w_{i}^{B W} \pi_{b+i, \lambda, c}(x)$,

where

$w_{i}^{B w}=w_{i}^{B W}(a, b)=\frac{(-1)^{j}}{(a+i) B(a, b)}\left(\begin{array}{c}b-1 \\ i\end{array}\right)$

By combining (15) and (13), we can easily write

$f_{B w}(x)=\sum_{j=0}^{\infty} t_{j}^{B W} \mathbf{g}_{j}, c(x)$.

where

Third, the linear combination for the GW density (9) follows from Castellares and Lemonte (2015) as 
$f_{G W}(x)=\sum_{i=0}^{\infty} w_{i}^{G W} \pi_{a+i, \lambda, c}(x)$,

where

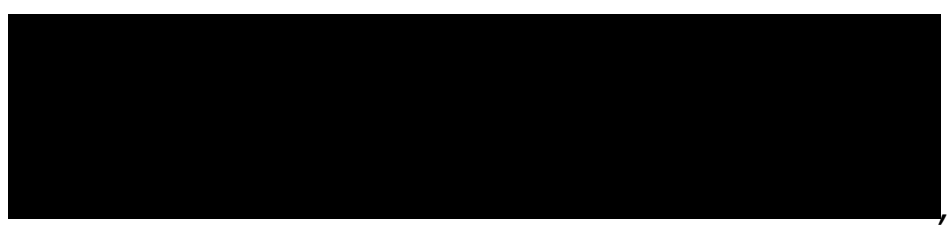

and $\psi_{i-1}(\cdot)$ are the Stirling polynomials given by

$$
\begin{aligned}
\psi_{h-1}(x)= & \frac{(-1)^{n-1}}{(n+1) !}\left[H_{n}^{n-1}-\frac{x+2}{n+2} H_{n}^{n-2}+\frac{(x+2)(x+3)}{(n+2)(n+3)} H_{n}^{n-3}-\cdots\right. \\
& \left.+(-1)^{n-1} \frac{(x+2)(x+3) \cdots(x+n)}{(n+2)(n+3) \cdots(2 n)} H_{n}^{0}\right]
\end{aligned}
$$

and $H_{n}{ }^{m}$ are positive integers given recursevily by

$$
H_{n+1}^{m}=(2 n+1-m) H_{n}^{m}+(n-m+1) H_{n}^{m-1} \text {, with }
$$

By combining (17) and (13), we obtain (for $a>0$ real non-integer)

$$
f_{G W}(x)=\sum_{j=0}^{\infty} t_{j}^{G W} g_{h_{j}, c}(x)
$$

where

For the last generalization, the KwW density (12) can be expressed (Nadarajah et al., 2012) as

$f_{K w W}(x)=\sum_{i=0}^{\infty} w_{i}^{K w W} \eta_{(i+1) a, \lambda, c}(x)$,

where

$$
w_{i}^{K w W}=w_{i}^{K w W}(\mathbf{b})=\frac{(-1)^{\mathrm{i}} \mathbf{b}}{(\mathbf{i}+1)}\left(\begin{array}{c}
\mathbf{b}-1 \\
i
\end{array}\right)
$$

By combining (19) and (13), we obtain 
$f_{K w W}(x)=\sum_{j=0}^{\infty} t_{j}^{k w W} g_{h_{j}, c}(x)$

where $t_{j}^{\mathrm{K}_{j} w W}=\sum_{i=0}^{\infty} w_{i}^{\mathrm{kwWW}} v_{j}((i+1)$ a) (for $\mathrm{j} \geq 0$ )

Equations (14), (16), (18) and (20) have identical linear combinations of Weibull densities with just different coefficients. They can be reduced to a simple equation

$$
f_{\star}(x)=\sum_{j=0}^{\infty} t_{j}^{\star} g_{j}, c(x)_{r}
$$

where $\star$ stands for any of the four generalized Weibull distributions. So, whenever possible, several mathematical properties for these generalized distributions can be determined in a similar manner from those Weibull properties.

\section{MATHEMATICAL PROPERTIES}

In this section, we present the main properties of the Weibull distribution which can be used in the linear representation (21) to obtain those properties for the MOW, $\mathrm{BW}, \mathrm{GW}$ and KwW distributions.

Some of the most important features of a distribution can be studied through moments. The $n$th ordinary moment of $T \sim \mathrm{W}(\lambda, c)$ is $E\left(T^{n}\right)=\lambda_{-}{ }^{n} \Gamma(n / c+1)$. Then, the moments of the four generalized Weibull distributions take the common form

$$
\mu_{\star, n}^{\prime}=\Gamma\left(\frac{n}{c}+1\right) \sum_{j=0}^{\infty} \frac{t_{i}^{*}}{\lambda_{j}^{n}}
$$

So, their cumulants $\left(K_{n}\right)$ can be found recursively from where

The skewness and kurtosis can be calculated from the third and fourth standardized cumulants.

The $n$th incomplete moment of is easily found changing variables from the lower incomplete gamma function as $m_{n}(y)=$ 
$\lambda-{ }^{n} y\left(n / c+1,(\lambda z)^{c}\right)$. Then, the incmomplete moments of the four generalized Weibull distributions reduce to

$$
\mathbf{m}_{\star, n}(z)=\sum_{j=0}^{\infty} \frac{t_{j}^{*}}{\lambda_{j}^{n}} \vee\left(\frac{n}{c}+1,\left(\lambda_{j} z\right)^{c}\right)
$$

The first incomplete moment $m_{*, 1}(z)$ is used to construct the Bonferroni and Lorenz curves (popular measures in economics, reliability, demography, insurance and medicine) and to determine the totality of deviations from the mean and median of a distribution.

For a given probability $\pi$, the Bonferroni and Lorenz curves of any of these four generalized

Weibull distributions are given by ) and $L_{\star}(\pi)=\pi B_{\star}(\pi)$, respectively, where $q=Q_{\star}(\pi)$ is the qf of the chosen distribution (Section 2)

The total deviations from the mean and median for any of these four generalizations can be expressed and $($ ) where w can be determined for the cdf chosen (Section 2).

We provide two expressions for the moment generating function (mgf) of $T$, say $M_{\lambda, c}(t)=E\left(\mathrm{e}^{\mathrm{tT}}\right)$. We can write

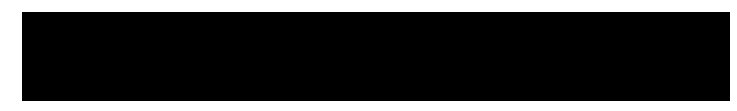

A first representation for $M_{\lambda, c}(t)$ is obtained from the Wright generalized hypergeometric function, namely

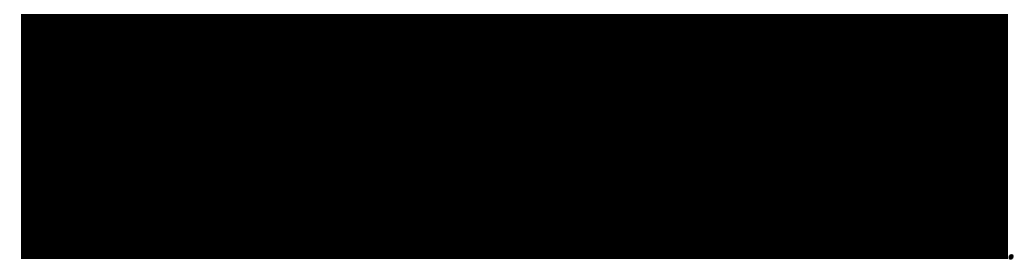

The Wright function exists if 1 0. For $c>1$, we have 


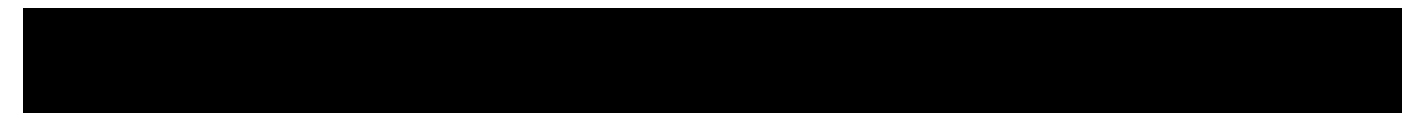

and then

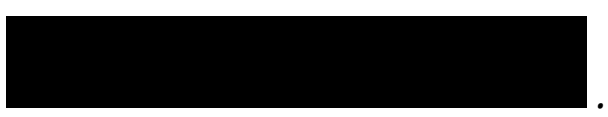

A second representation for $M(t)$ is based on the Meijer G-function defined by

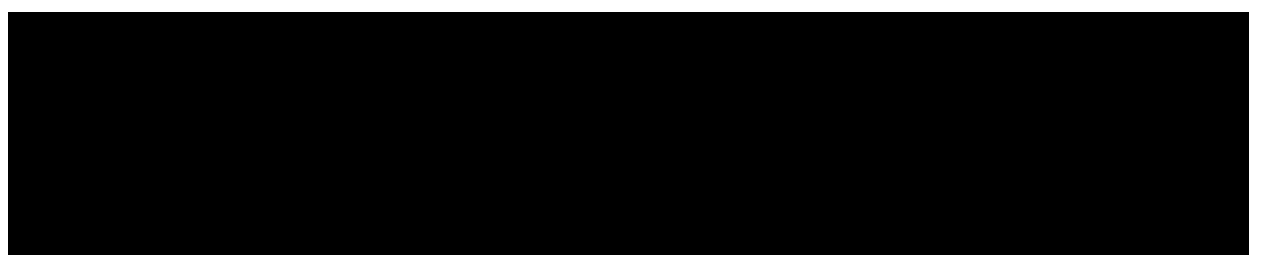

where $\mathrm{i}=\sqrt{ }-1$ is the complex unit and $L$ denotes an integration path (see Section 9.3 in Gradshteyn and Ryzhik (2000) for a description of this path). The Meijer Gfunction contains many integrals with elementary and special functions (Prudnikov et al., 1986). For an arbitrary function $g(\cdot)$, based on the result

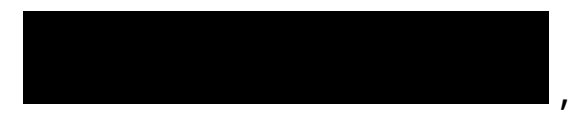

we can write $M_{\lambda, c}(t)$ as

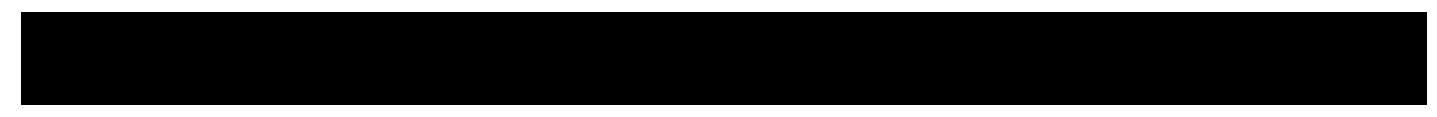

We now assume that $c=p / q$, where $p \geq 1$ and $q \geq 1$ are co-prime integers. Note that this condition for calculating the integral $M_{\lambda, c}(t)$ is not restrictive since every real number can be approximated by a rational number. Using equation (2.24.1.1) in Prudnikov et al. (1986, volume 3), we obtain

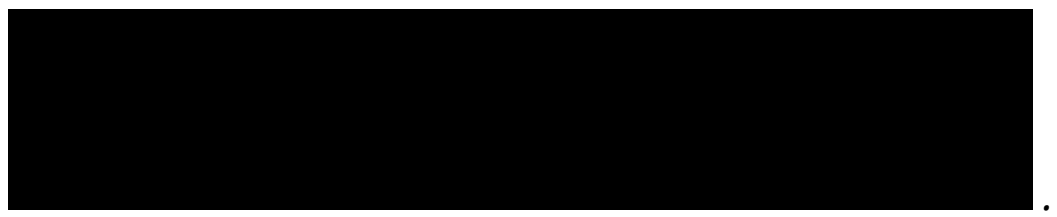


Then, the generating functions of the four generalized distributions can be written as

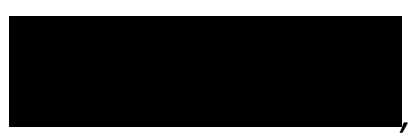

where $M_{\lambda, c}(t)$ follows from the two expressions for $M_{\lambda, c}(t)$ given before with $\lambda=\lambda_{j}$.

\section{OTHER EXTENDED WEIBULL DISTRIBUTIONS}

A first distribution closely related to the EW distribution is the generalized Weibull distribution pioneered by Mudholkar et al. (1996) with cdf (for $x>0$ )

$$
F(x)=1-\left[1-G(\lambda x)^{1 / c}\right]^{1 / s}
$$

where $\lambda>0, c>0$ and $a>0$. This distribution allows for bathtub shaped, monotonically increasing, monotonically decreasing and constant hazard rates.

Gera (1997) presented a modification of the EW cdf given by $F_{*}(x)=$ $\exp \left(-s x^{\prime}\right) G_{\lambda, c}(x)^{a}$ for $r>0$ and $s>0$. Lai et al. (2003) introduced the modified Weibull (MW) density (for $x>0) f(x)=a(c+\delta x) x^{c}{ }_{-1}^{1} \exp (\delta x) \exp \left[-a x^{c} \exp (\delta x)\right]$, where $a>0, c>0$ and $\delta>$ 0 , which allows for increasing and bathtub shaped hazard rates.

Nadarajah and Kotz (2005) proposed a four-parameter generalized WeibullGompertz distribution with cdf (for $x>0$ )

$$
F(x)=1-\exp \left\{-\alpha x^{c}\left\{\exp \left(\beta x^{d}\right)-1\right]\right\}, \quad \alpha, \beta, c \geq 0, d>0
$$

Again, this cdf can lead to increasing, decreasing or bathtub-shaped hazard rates.

Bebbington et al. (2007) defined the flexible Weibull density (for $x>0$ ) as

$$
F(x)=1-\exp \{-[\exp (\alpha x-\beta / x)]\}, \quad \alpha, \beta>0 .
$$


Carrasco et al. (2008) defined the generalized modified Weibull (GMW) density (for $x>0)$ as

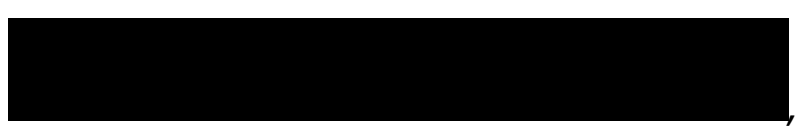

where $a>0, y \geq 0, \lambda \geq 0$ and $\beta>0$.

Nikulin and Haghighi (2009) proposed the cdf of the power generalized Weibull distribution (for $x>0$ ) as $F(x)=1-\exp \left\{1-\left[1+(\lambda x)^{v}\right]^{1 / \eta}\right\}$, where $v>0, y \geq 0$ and $\lambda>0$, which allows for increasing, decreasing, constant, bathtub shaped, and unimodal hazard rates.

Further, Barreto-Souza et al. (2010) defined the four-parameter beta generalized exponential (BGE) density (for $x>0$ ) as

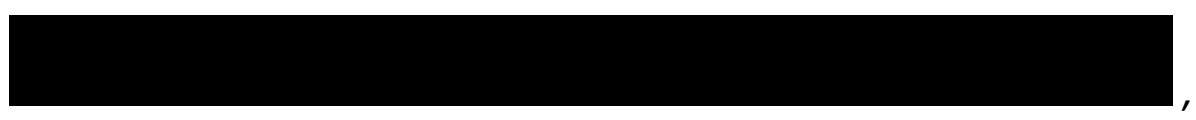

where $a>0, b>0, a>0$ and $\lambda>0$. The BGE model allows for bathtub shaped, monotonically increasing, monotonically decreasing and upside-down bathtub hazard rates.

Silva et al. (2010) introduced the five-parameter beta modified Weibull (BMW) density (for $x>0$ )

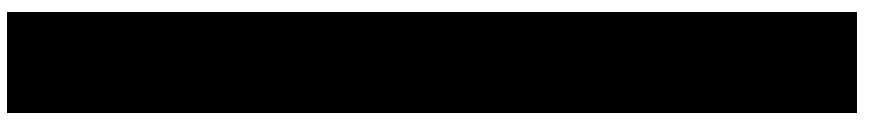

where $a>0, b>0, a>0, y>0$ and $\lambda \geq 0$. The BMW distribution includes as submodels seventeen known distributions such as the EW, BW, MW and GMW distributions, among others. It allows for bathtub shaped, monotonically decreasing, monotonically increasing, and upside down bathtub shaped hazard rates.

Pascoa et al. (2011) defined the five-parameter Kumaraswamy-generalized gamma density (for $x>0$ ) 


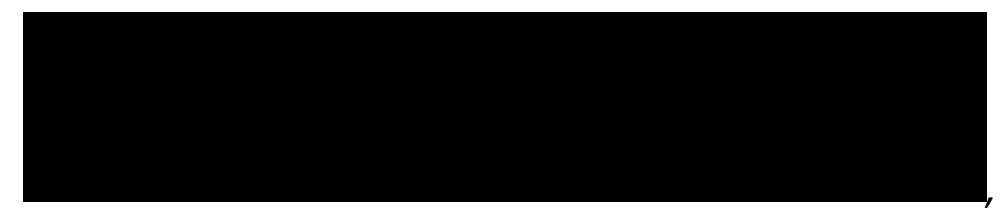

where $y_{1}(p, x)=\gamma(p, x) / \Gamma(p), a>0, \lambda>0, k>0, \psi>0$ and $\tau>0$. This distribution contains as special cases the exponentiated generalized gamma (Cordeiro et al., 2011b) and KwW distributions, among several others. It allows for constant, bathtub shaped, monotonically decreasing, monotonically increasing, and upside down bathtub shaped hazard rates.

Cordeiro et al. (2011c) proposed the beta extended Weibull family density (for $x>$ 0) by

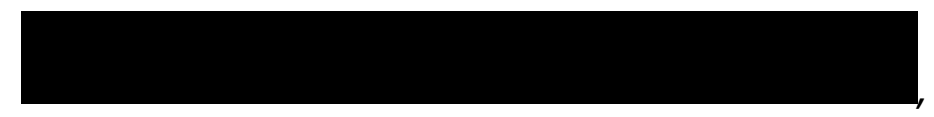

where $a>0, b>0$ and $a>0, H(x) \geq 0$ is a monotonic increasing function of $x$ and $h(x)=d H(x) / d x$. This family contains as sub-models the EW and BMW distributions and also give rise to many new classes of distributions.

Finally, Alexander et al. (2012) proposed a class of generalized beta-generated densities given by $f_{*}(x)=c g(x) G(x)^{a c} c^{1}\left[1-G(x)^{c}\right]^{b}{ }_{-}^{1} / B(a, b)$ for shape parameters $a>0, b>$ 0 and $c>0$, where $G(\cdot)$ is a valid cdf and $g(\cdot)$ is its pdf. Two important special cases are the beta and Kumaraswamy generated families discussed in Section 2.

\section{MAXIMUM LIKELIHOOD ESTIMATION}

The parameters of the MOW, BW, GW and KwW distributions are estimated by maximum likelihood from complete samples only. Let $x_{1}, \cdots, x_{n}$ be a random sample of size $n$ from the distributions given by (4), (6), (8) and (12) and parameter vector $\eta$. Let $q$ denote the dimension of $\eta$. Parametric inference for such data is usually based on likelihood methods and asymptotic theory. The log-likelihoods $(/(\eta))$ for the model parameters are 
-For the MOW distribution, $\eta=(\lambda, c, \theta)^{T}$

-For the BW distribution, $\eta=(\lambda, c, a, b)^{T}$

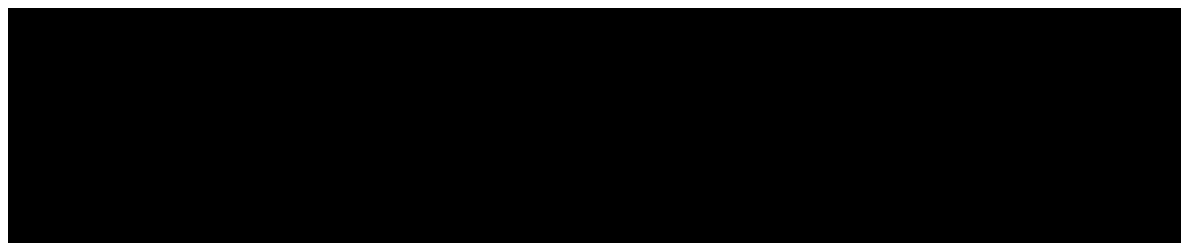

-For the GW distribution, $\eta=(\lambda, c, a)^{T}$

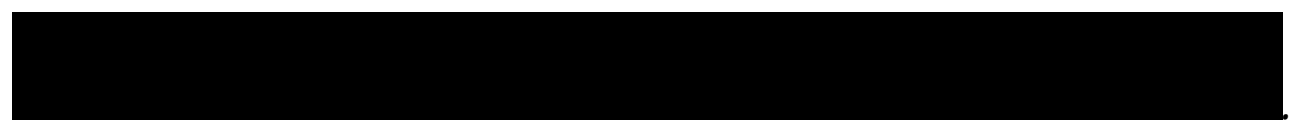

-For the KwW distribution, $\eta=(\lambda, c, a, b)^{T}$

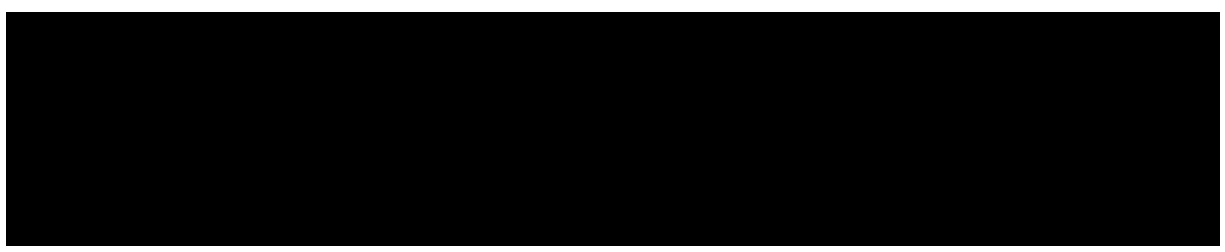

where $G_{\lambda, c}\left(X_{i}\right)$ and $g_{\lambda, c}\left(X_{i}\right)$ are defined in Section 1.

The function $/(\eta)$ can be maximized either directly by using well-known platforms such as the R (optim function), SAS (PROC NLMIXED), Ox program (MaxBFGS sub-routine) or by solving the nonlinear likelihood equations $\partial /(\eta) / \partial \eta=\mathbf{0}$. These equations cannot be solved analytically numerically. We can use iterative techniques such as the Newton-Raphson type algorithms withb and statistical software can be used to obtain the maximum likelihood estimate (MLE) $\eta$ of $\eta$

initial values for the parameters taken from the fitted Weibull distribution.

For interval estimation and hypothesis tests on the model parameters, we require the observed information matrix since its expectation requires numerical integration. Under standard regularity conditions that are fulfilled for parameters in 
the interior of the parameter space but not on the boundary, the asymptotic distribution of

$$
(\hat{\eta}-\eta) \text { is } N_{q}\left(0, I(n)^{-1}\right) \text {, }
$$

where $I(\eta)$ is the expected information matrix. This asymptotic behavior is valid if $I(\eta)$ is $\operatorname{reN}_{q}\left(\mathbf{0}, J(\eta)-^{1}\right)$ b distribution can be used to construct approximate confidence intervals and confib placed by $J(\eta)$, i.e., the observed information matrix evaluated at $\eta$. The multivariate normal dence regions for the individual parameters and for the hazard rate and survival functions.

\section{APPLICATIONS}

All generalized distributions mentioned previously can be fitted to real data sets using the AdequacyModel package by the BFGS method in the R software.

In this section, we provide two applications comparing the four generalized distributions (MOW, BW,GW, KwW) by means of two real data sets. We calculate the MLEs of the parameters for the fitted distributions, their standard errors (SES) (in parentheses) and the following goodness-of-ft measures: the Akaike Information Criterion (AIC), Bayesian Information Criterion (BIC), Cramervon Misses (W*) statistic and Anderson Darling $\left(A^{*}\right)$ statistic using the AdequacyModel package. The better fits to the data correspond to small values of these measures.

\subsection{Application 1: Repair time data}

The following maintenance data refer to active repair times (in hours) for an airborne communication transceiver. The 45 repair times analyzed by Chhikara and Folks (1977) are: $0.2,0.3,0.5,0.5,0.5,0.5,0.6,0.6,0.7,0.7,0.7,0.8,0.8,1.0,1.0,1.0,1.1$, 1.3, 1.5, 1.5, 1.5, 1.5, 2, 2, 2.2, 2.5, 2.7, 3.0, 3.0, 3.3, 3.3, 4.0, 4.0, 4.5, 4.7, 5.0, 5.4, 5.4, $7.0,7.5,8.8,9.0,10.3,22.0,24.5$. 
The results from some fitted distributions to these data are listed in Table 1. We can note that the KwW model is the most adequate distribution to these data. The four statistics are favorable when compared to the nested Weibull distribution. For non-nested models, the statistics $W_{*}$ and $A_{*}$ are favorable to the KwW model with the smallest values.

Table 1 - MLEs of the model parameters for the repair time data, their SEs (given in parentheses) and statistical measures

\begin{tabular}{|c|c|c|c|c|c|c|c|c|}
\hline Model & $c$ & $\lambda$ & $a$ & $b$ & AIC & BIC & $W_{*}$ & $A *$ \\
\hline \multirow[t]{2}{*}{ BW } & 0.591 & 8.849 & 7.964 & 0.227 & 207.39 & 214.70 & 0.041 & 0.282 \\
\hline & $(0.179)$ & $(0.051)$ & $(2.311)$ & $(0.209)$ & & & & \\
\hline \multirow[t]{2}{*}{ KwW } & 0.597 & 9.999 & 11.830 & 0.200 & 206.86 & 214.17 & 0.037 & 0.256 \\
\hline & $(0.177)$ & $(0.358)$ & $(4.667)$ & $(0.188)$ & & & & \\
\hline \multirow[t]{2}{*}{ GW } & 0.457 & 8.022 & 4.041 & - & 209.39 & 214.87 & 0.081 & 0.551 \\
\hline & $(0.060)$ & (7.543) & (1.083) & $(-)$ & & & & \\
\hline \multirow[t]{2}{*}{ EW } & 0.545 & 1.198 & 3.260 & - & 208.78 & 214.26 & 0.075 & 0.509 \\
\hline & $(0.121)$ & $(0.928)$ & $(1.760)$ & $(-)$ & & & & \\
\hline \multirow[t]{2}{*}{ Weibull } & 0.898 & 0.294 & 1 & 1 & 212.93 & 216.59 & 0.129 & 0.900 \\
\hline & $(0.095)$ & $(0.051)$ & $(-)$ & $(-)$ & & & & \\
\hline Model & $c$ & $\lambda$ & $\boldsymbol{\theta}$ & & AIC & BIC & $W *$ & $A *$ \\
\hline \multirow[t]{2}{*}{ MOW } & 1.484 & 0.053 & 0.033 & & 207.71 & 213.19 & 0.069 & 0.438 \\
\hline & $(0.204)$ & $(0.043)$ & $(0.051)$ & & & & & \\
\hline
\end{tabular}

The histogram and the plots of the fitted KwW and Weibull densities are displayed in Figure 1a. The plots of the empirical survival and estimated survivals are displayed in Figure $1 \mathrm{~b}$. The plots of the empirical hazard and estimated hazards are displayed in Figure 1c. We conclude from these plots that the KwW distribution is very suitable to these data. 


\subsection{Application 2: Chlorobenzene data}

In this application, the data refer to a sample with chlorobenzene concentrations in the air of a factory (in TWAs/15min). The 31 observations represent the average concentrations calculated every fifteen minutes in an eight-hour shift (Kumagai and Matsunaga, 1995). The averages are: 13.7, 10.2, 9.9, 4.3, 5.6, 45.6, 42.0, 14.1, 3.8, 9.3, 10.6, 91.3, 2.2, 3.8, 6.0, 17.8, 131.8, 31.0, 4.2, 2.6, 27.6, 1.7, 7.0, 2.1, 1.5, $7.5,2.5,2.4,51.9,12.9,12.3$.

Figure 1 - Plots for repair time data. (a) Estimated KwW and Weibull densities. (b) Estimated survivals of the KwW and Weibull distributions. (c) Estimated hazards of the KwW and Weibull distributions

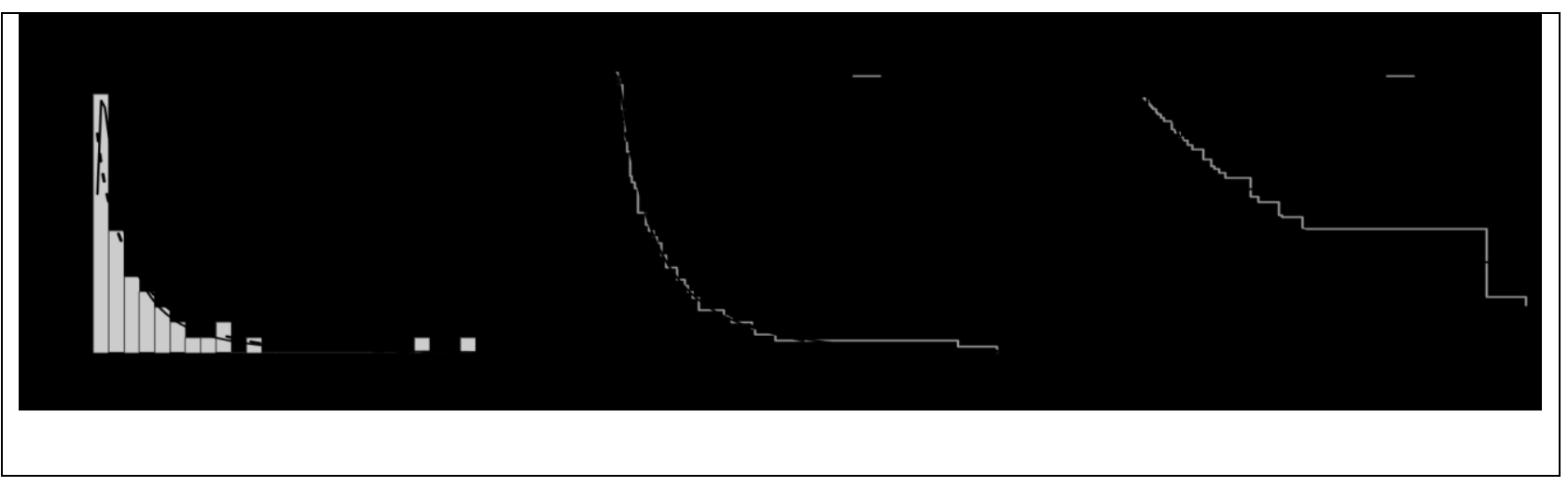

The results from some fitted distributions to these data are listed in Table 2. We conclude that the KwW model is the most suitable to these data. The four statistics are favorable when compared to the nested Weibull distribution. For non-nested models, the measures $W_{*}$ and $A_{*}$ are favorable for the KwW model with the smallest values. 
Table 2 - MLEs of the model parameters for the chlorobenzene data, their SEs (given in parentheses) and statistical measures

\begin{tabular}{|c|c|c|c|c|c|c|c|c|}
\hline Model & c & $\lambda$ & $a$ & $\boldsymbol{b}$ & AIC & BIC & $W *$ & $A *$ \\
\hline \multirow[t]{2}{*}{ BW } & 0.558 & 3.217 & 11.843 & 0.181 & 241.23 & 246.96 & 0.039 & 0.291 \\
\hline & $(0.072)$ & $(0.096)$ & (5.373) & $(0.085)$ & & & & \\
\hline \multirow[t]{2}{*}{ KwW } & 0.532 & 5.037 & 27.757 & 0.161 & 240.19 & 245.92 & 0.033 & 0.248 \\
\hline & $(0.026)$ & $(0.028)$ & $(0.001)$ & $(0.040)$ & & & & \\
\hline \multirow[t]{2}{*}{ GW } & 0.316 & 38.670 & 6.854 & - & 242.76 & 247.06 & 0.078 & 0.539 \\
\hline & (0.019) & $(0.002)$ & $(0.793)$ & $(-)$ & & & & \\
\hline \multirow[t]{2}{*}{ EW } & 0.284 & 8.949 & 21.677 & - & 240.02 & 244.33 & 0.042 & 0.319 \\
\hline & $(0.080)$ & $(0.493)$ & (3.100) & $(-)$ & & & & \\
\hline \multirow[t]{2}{*}{ Weibull } & 0.820 & 0.059 & 1 & 1 & 245.95 & 248.82 & 0.149 & 0.943 \\
\hline & $(0.106)$ & $(0.013)$ & $(-)$ & $(-)$ & & & & \\
\hline Model & c & $\lambda$ & $\boldsymbol{\theta}$ & & AIC & BIC & $W *$ & $A *$ \\
\hline \multirow[t]{2}{*}{ MOW } & 1.416 & 0.008 & 0.025 & & 241.6 & 245.90 & 0.052 & 0.394 \\
\hline & $(0.224)$ & $(0.006)$ & $(0.035)$ & & & & & \\
\hline
\end{tabular}

The histogram and the plots of the fitted KwW and Weibull densities are displayed in Figure 2a. The plots of the empirical cdf and estimated cdfs are given in Figure $2 \mathrm{~b}$. The plots of the empirical hazard and estimated hazards are displayed in Figure $2 \mathrm{c}$. We conclude from these plots that the KwW distribution is very suitable to these data. 
Figure 2 - Plots for chlorobenzene data. (a) Estimated KwW and Weibull densities. (b) Estimated cdfs of the KwW and Weibull distributions. (c) Estimated hazards of the KwW and Weibull distributions

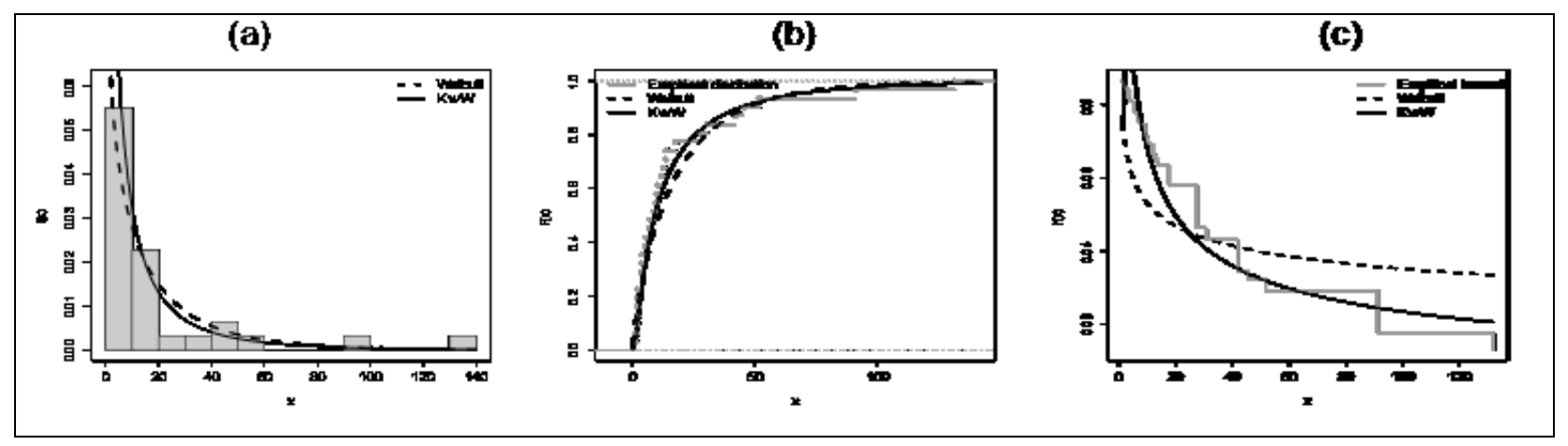

\section{CONCLUSIONS}

In this paper, we present a simple linear representation which holds for the densities of various extensions of the Weibull distribution. It involves coefficients that depend only on the parameters of the families and the Weibull densities in convenient forms. We demonstrate the usefulness of this representation for the he MarshallOlkin-Weibull, beta-Weibull, gamma-Weibull and Kumaraswamy-Weibull distributions. However, it can be valid for some other generalized Weibull distribution. The main advantage of the linear representation is to derive easily some mathematical properties for these four distributions from those Weibull properties. We present two applications of these generalized distributions to real data sets.

\section{REFERENCES}

Alexander, C., Cordeiro, G. M., Ortega, E. M. M. and Sarabia, J. M. (2012). Generalized betagenerated distributions. Computational Statistics and Data Analysis, 56, 1880-1897.

Barreto-Souza, W., Santos, A. and Cordeiro, G. M. (2010). The beta generalized exponential distribution. Journal of Statistical Computation and Simulation, 80, 159-172.

Bebbington, M., Lai, C.D. and Zitikis, R. (2007). A flexible Weibull extension. Reliability Engineering and System Safety, 92, 719-726. 
Carrasco, J. M. F., Ortega, E. M. M. and Cordeiro, G. M. (2008). A generalized modified Weibull distribution for lifetime modeling. Computational Statistics and Data Analysis, 53, 450-462.

Castellares, F. and Lemonte, A. (2015). A new generalized Weibull distribution generated by gamma random variables. Journal of the Egyptian Mathematical Society, 23, 382-390.

Chhikara, R. S. and Folks, J. L. (1977). The Inverse Gaussian Distribution as a Lifetime Model. Technometrics, 19, 461.

Cordeiro, G. M. and de Castro, M. (2011). A new family of generalized distributions. Journal of Statistical Computation and Simulation, 81, 883-898.

Cordeiro, G. M., Ortega, E. M. M. and Silva, G. (2011b). The exponentiated generalized gamma distribution with application to lifetime data. Journal of Statistical Computation and Simulation, 81, 827-842.

Cordeiro, G. M., Ortega, E. M. M. and Silva, G. (2011c). The beta extended Weibull family. Journal of Probability and Statistical Science, 10, 15-40.

Cordeiro, G. M., Lemonte, A. and Ortega, E. M. M. (2014). The Marshall-Olkin Family of Distributions: Mathematical Properties and New Models. Journal of Statistical Theory and Practice, 8, 343-366.

Cordeiro, G. M., Lemonte, A. and Ortega, E. M. M. (2014). The Marshall-Olkin family of distributions: Mathematical properties and new models. Journal of Statistical Theory and Practice, 8, 343-366.

Eugene, M, Lee, C. and Famoye, F. (2002). Beta-normal distribution and its applications. Communication in Statistics - Theory Methods, 31, 497-512.

Gera, A. W. (1997). The modified exponentiated-Weibull distribution for lifetime modeling. In: 1997 Proceedings of the Annual Reliability and Maintainability Symposium, pp. 149-152.

Kumagai, S. and Matsunaga, I. (1995). Changes in the distribution of short-term exposure concentration with different averaging times. American Industrial Hygiene Association Journal, 56, 24- 31.

Lai, C. D., Xie, M. and Murthy, D. N. P. (2003). A modified Weibull distribution. IEEE Transactions on Reliability, 52, 33-37.

Marshall, A. W., Olkin, I. (1997). A new method for adding a parameter to a family of distributions with application to the exponential and Weibull families. Biometrika, 84, 641652. 
Mudholkar, G. S. and Srivastava, D. K. (1993). Exponentiated Weibull family for analyzing bathtub failure-rate data. IEEE Transactions on Reliability, 42, 299-302.

Mudholkar, G. S. and Hutson, A. D. (1996). The exponentiated Weibull family: some properties and a flood data application. Communications in Statistics-Theory and Methods, 25, 3059-3083.

Nadarajah S. and Kotz S. (2005). On some recent modifications of Weibull distribution. IEEE Transactions on Reliability, 54, 561-562.

Nadarajah, S., Cordeiro, G. M., and Ortega, E. M. M. (2012). General results for the Kumaraswamy $G$ distribution. Journal of Statistical Computation and Simulation, 82, 951979.

Nikulin, M. and Haghighi, F. (2009). On the power generalized Weibull family. Metron, 67, 75-86.

Pascoa, M. A. P., Ortega, E. M. M. and Cordeiro, G. M. (2011). The Kumaraswamygeneralized gamma distribution with application in survival analysis. Statistical Methodology, 8, 411-433.

Prudnikov, A. P., Brychkov, Y. A. and Marichev, O. I. (1986). Integrals and Series, volumes 1, 2 and 3. Gordon and Breach Science Publishers, Amsterdam.

Silva, G. O., Ortega, E. M. M. and Cordeiro, G. M. (2010). The beta modified Weibull distribution. Lifetime Data Analysis, 16, 409-430.

Stacy, E. W. (1962). A generalization of the gamma distribution. Annals of Mathematical Statistics, 33, 1187-1192.

Zografos, K. and Balakrishnan, N. (2009). On families of beta- and generalized gamma generated distributions and associated inference. Statistical Methodology, 6, 344-362. 